\title{
Association between a history of child neglect, abuse and the suicidal patient: a cross-sectional study
}

\author{
Ali Delirrooyfard ${ }^{1 *}{ }^{*}$ Maria Cheraghi $^{(\oplus}{ }^{\circledR}$ Mehdi Sayyah $^{3 \oplus}$, Zahra Farahbakhsh $^{4}$ \\ 'Imam Khomeini Hospital Clinical Research Development Unit, Ahvaz Jundishapur University of Medical Sciences, \\ Ahvaz, Iran \\ ${ }^{2}$ Department of Public Health, Ahvaz Jundishapur University of Medical Sciences, Ahvaz, Iran \\ ${ }^{3}$ Department of Psychiatry, Ahvaz Jundishapur University of Medical Sciences, Ahvaz Iran \\ ${ }^{4}$ Student Research Committee, Ahvaz Jundishapur University of Medical Sciences, Ahvaz, Iran
}

\author{
Received: 3 September 2021 \\ Accepted: 22 November 2021 \\ Published online: 7 December 2021 \\ *Corresponding author: \\ Ali Delirrooyfard, \\ Imam Khomeini Hospital Clinical \\ Research Development Unit, Ahvaz \\ Jundishapur University of Medical \\ Sciences, Ahvaz, Iran \\ Tel: +989173159418 \\ Email: adelir2891@gmail.com \\ Competing interests: None. \\ Funding information: None. \\ Citation: Delirrooyfard A, Cheraghi \\ M, Sayyah M, Farahbakhsh Z. \\ Association between a history of \\ child neglect, abuse and the suicida \\ patient: a cross-sectional study. \\ Journal of Emergency Practice \\ and Trauma 2022; 8(1): 32-36. doi \\ 10.34172/jept.2021.34
}

\begin{abstract}
Objective: People with a significant childhood history of abuse may exhibit emotional dysregulations and psychiatric disorders and, in some cases, present suicidal ideation. Methods: In this descriptive-analytical and cross-sectional study data were collected from suicidal patients referred to two grand hospitals in 2019. One hundred ninetyfive participants were evaluated concerning child abuse and neglect history. Data were reported as mean, SD, frequency, and percent. T-test and chi-square tests were used for statistical analyses accordingly.

Results: Child abuse regarding suicidal people were to the following order, neglect $(n=103)$, child emotional abuse $(n=102)$, child physical abuse $(n=101)$, malnutrition $(n=96)$, and child sexual abuse $(n=87)$. Suicide was different between the two genders, but it was not different based on parents' education and marital status.

Conclusion: The harassed child is not equipped with proper behavioral skills and is subjected to low self-esteem due to incorrect training. Therefore, he/she may commit suicide in adolescence by facing some problems and failures. In this regard, community plays an important role, parents must be informed and use the right culture to reward the child.

Keywords: Suicide, Child abuse, Child neglect, Family characteristics
\end{abstract}

\section{Introduction}

Suicidal behavior is a range of behaviors that involves thinking about or acting on self-destruction. Suicide risk factors include psychiatric disorders, especially depression, anxiety and eating disorders, old age, marital status, education, job status, and male gender (1-4). Suicidal behavior is a spectrum that includes thinking about suicide to acting on it. Some patients have suicidal thoughts that they never do it. Some patients plan days, weeks, or even years before attempting suicide, and some patients commit suicide because of a sudden, thoughtless motive (5).

Any behavior or failure to perform an action that endangers the child's physical, mental, and developmental health is called child abuse (6). Experts believe that any physical, psychological, sexual abuse or exploitation and failure to address the human needs of persons under 18 is considered child abuse by other people $(7,8)$. Child abuse is divided into physical abuse, emotional abuse, and sexual abuse (9-11). Symptoms of childhood abuse include mood swings and isolationism, anxiety, difficulty in sleeping, nail-biting, academic failure, nightmares, abnormal behaviors, fear of touch, unrelenting anger at parents, and fears so far that have not yet existed $(12,13)$. Studies have shown that the cerebral cortex of those who experience sexual trauma is thinner than healthy people. Hence, their analysis and decision-making power are logical. These people are disadvantaged in adulthood and cannot adapt easily to the world around them $(14,15)$. Many neurobiological studies have shown that childhood abuse may affect the prefrontal cortex, thereby disrupting inhibitory and motivational-fear response systems. Some of these findings correlate with the neurobiological features of impulsivity and suicide incidence (6). Community health care providers, especially those responsible for assessing people with mental health problems, need to be aware of the relationship between child abuse types and their specific psychological conditions (16). Such conditions provide the basis for committing violence against themselves and others and increase the risk of 
suicide in these individuals $(17,18)$. According to studies conducted, child abuse prevention can significantly reduce the prevalence of mental disorders and violent behaviors, especially suicide in adolescents and adults (19). Given the importance of determining the prevalence, relationship, and recognition of mental disorders in people with a history of child abuse, this study aimed to determine the prevalence of these disorders and identify related factors.

\section{Methods}

This descriptive-analytical and cross-sectional study was limited to a population who attempted suicide and were admitted into two Grand hospitals in Ahvaz city in 2019. All participants in the study filled out the informed consent form and the Ethics Committee of Ahvaz Jundishapur University of Medical Sciences approved this study by code number IR. AJUMS.REC.1396.1000 and patients were not forced to enter the study. No invasive procedure was performed, and all the processes of the study and questionnaire completion were explained to the patients. The number of the sample was equal to 195 people.

Study data included two categories of demographic variables and the main variables (child abuse). The questionnaire consisted of different dimensions of child abuse, and based on the completed questionnaires, the history of exposure to child abuse was studied and evaluated. The study questionnaire was developed based on 25 questions aimed at assessing child abuse in different dimensions, including child abuse, child sexual abuse, neglect, malnutrition, and child emotional abuse. The reliability of the questionnaire was calculated by Cronbach's alpha method for the different dimensions of the questionnaire in Maniglio 2011 and it was 0.80 (20). To determine the validity and reliability, we calculated the items making up the intensity and content of these variables. The results showed a very high correlation between each item with intensity and content at a significance level above 95\% (21). The questions were Likert-type, with a rating of "very low $=25$, low $=50$, average $=75$, high $=100$, and very high $=125$ ". The interpretation of the questionnaire is based on the response score of "25-50: low child abuse", "50-75: moderate child abuse, and above 75: high child abuse. Cronbach's alpha coefficient in the present study was 0.83 .

The inclusion criterion encompassed people who were willing to participate in the study, and the exclusion criterion were people who did not consent to participate in the study. All descriptive data were reported as mean, standard deviation, distribution tables, and frequency percent. Data were analyzed using SPSS software version 20 and $t$ test and chi-square tests were used accordingly. $P$ value $<0.05$ was considered significant.

\section{Results}

The results obtained from the preliminary analysis of demographic variables show that the mean age of suicide was $17.96 \pm 6.3$ years. Most patients $(38.46 \%)$ were in the range of 15-25 years, but the prevalence of suicide was not significantly different between the age groups $(P=0.235)$. The prevalence of suicide was significantly higher among women $(P=0.024)$. Generally, the suicide attempt was in patients whose parents had an emotional divorce (40.51\%), but there was no significant difference between patients with different parents' marital status $(P=0.102)$. Suicide in participants whose parents had an associate degree was higher than others $(56.41 \%$ of fathers and $59.49 \%$ of mothers), but there was no significant difference between patients with parents of different educational levels $(P=0.079)$ (Table 1$)$.

According to the results, the domains of child abuse in the suicidal people on average score were almost related to: neglect (103/125), child emotional abuse (102/125), child physical abuse (101/125), malnutrition (96/125), and child sexual abuse (87/125) (Table 2).

Regarding the results, child physical abuse experienced

Table 1. Demographic information of patients

\begin{tabular}{|c|c|c|}
\hline & No. $(\%)$ & $P$ value \\
\hline \multicolumn{3}{|l|}{ Age $(y)$} \\
\hline$<15$ & $34(17.44)$ & \multirow{5}{*}{0.235} \\
\hline $15-25$ & $75(38.46)$ & \\
\hline $26-35$ & $51(26.15)$ & \\
\hline $36-45$ & $21(10.77)$ & \\
\hline$>45$ & $14(7.18)$ & \\
\hline \multicolumn{3}{|l|}{ Gender } \\
\hline Female & $132(67.69)$ & \multirow[t]{2}{*}{0.024} \\
\hline Male & $63(32.31)$ & \\
\hline \multicolumn{3}{|l|}{ Marital status of parents } \\
\hline One of the parents died & $12(6.15)$ & \multirow{6}{*}{0.102} \\
\hline Parental separation & 43 (22.05) & \\
\hline Emotional divorce & $79(40.52)$ & \\
\hline Co-living & $38(19.48)$ & \\
\hline Having stepmother & $8(4.10)$ & \\
\hline Having stepfather & $15(7.69)$ & \\
\hline \multicolumn{3}{|l|}{ Parents' educational level } \\
\hline \multicolumn{3}{|l|}{ Diploma and less } \\
\hline Father & $50(25.64)$ & \multirow{8}{*}{0.079} \\
\hline Mother & $62(31.79)$ & \\
\hline Post-diploma and bachel & & \\
\hline Father & $110(56.41)$ & \\
\hline Mother & $116(59.49)$ & \\
\hline \multicolumn{2}{|l|}{ Master degree and above } & \\
\hline Father & 35 (17.95) & \\
\hline Mother & $17(8.72)$ & \\
\hline
\end{tabular}


Table 2. Different domains of child abuse

\begin{tabular}{|c|c|c|c|c|c|c|}
\hline \multirow{2}{*}{ Overall Score, Mean \pm SD } & & \multicolumn{5}{|c|}{ Participants Responses, No. (\%) } \\
\hline & & Very low & Low & Medium & High & Very High \\
\hline Physical & $101.75 \pm 24.5$ & $34(17.4)$ & $33(16.9)$ & $17(8.7)$ & $67(34.4)$ & $44(22.6)$ \\
\hline Sexual & $87.75 \pm 29.7$ & $48(24.6)$ & $45(23.1)$ & $8(4.1)$ & $56(28.7)$ & $38(19.5)$ \\
\hline Malnutrition & $96 \pm 27$ & $44(22.6)$ & $44(22.6)$ & $12(6.1)$ & $52(26.7)$ & $43(22.0)$ \\
\hline Neglect & $103.5 \pm 23$ & $36(18.4)$ & $38(19.5)$ & $6(3.1)$ & $70(35.9)$ & $45(23.1)$ \\
\hline Emotional & $102.75 \pm 25.5$ & $30(15.4)$ & $38(19.4)$ & $6(3.1)$ & $60(30.8)$ & $61(31.3)$ \\
\hline
\end{tabular}

Very Low $=25$, Low $=50$, Average $=75$, High $=100$, Very High $=125$

in $61 \%$ of participants, neglect in $65.5 \%$, child emotional abuse in $62 \%$, malnutrition in $36.4 \%$, and child sexual abuse in $31 \%$ of participants have high scores. (Table 3 ).

Concerning physical abuse, the history of whipping, and corporal punishment for not doing homework had the highest average. In sexual abuse, the most leading was related to rape by family members and acquaintances. In negligence, the highest average was the angry parents' inattention and lack of financial and social securement. In malnutrition, lack of proper nourishment compared to peers and the prohibition of eating as the punishment was the most. Finally, in emotional abuse, the family's lack of parental encouragement, rejection, and humiliation had the highest average.

\section{Discussion}

Child abuse is a severe social harm that can cause irreversible damage to the child's body and mind. Like any other social problems, it can be prevented and controlled by awareness and culture. This descriptive cross-sectional study determined the suicide rate despite a history of child abuse in suicidal individuals referred to the emergency department in 2019. The results show that suicide was not significantly different between age groups. In addition, $26.15 \%$ of people who committed suicide were in the age group of 15 to 25 years. This group has the most significant risks for suicidal behavior and dying, as reported by the WHO (22). In this study, suicide was significantly different between the two genders, but it was not significantly different based on parent's education.

Regarding the difference between the ratio of men and women concerning abuses, a study conducted in 2015 revealed that delinquent females were often prey to sexual and physical abuse and had a background of inattention in comparison to males. Besides, the association between victimization and violation was more probable in males than in females (23). More than half of the parents of those who committed suicide experienced an emotional divorce. The statistical analysis showed that suicide did not differ statistically between individuals based on their parents' marital status.

Neglect is the most prevalent kind of child abuse and can lead to severe and long-term injury and even death. Inattention ranges from leaving the child alone at home to malnutrition (24). In 2019, a study looked at parental neglect from two representatives of U.S. psychological evaluation showing that $6 \%$ of participants experienced parental neglect in the past year and $17 \%$ at some point in their lives. The subtype "lack of supervision to children" due to parents' absence was the highest. It has also been reported that all individuals with the experience of neglect had symptoms of self-harm, suicidal ideation, or increased risk of underage alcohol and illegal narcotic use (25). Afifi et al in Canada asserted that all forms of child abuse, including physical and sexual abuse and exposure to intimate partner violence were all strongly associated with unstable mental disorders, including suicidal ideation or suicide attempts. They also reported that all of these disorders and mental crises were higher in women than men (17). These events emphasize the importance of prevention approaches that provide the support essential to make reliable and healthy relationships and situations for children to thrive.

More than $70 \%$ of people who presented with a diagnosis

Table 3. The interpretation of different domains of child abuse questionnaire

\begin{tabular}{|c|c|c|c|c|}
\hline & \multicolumn{3}{|c|}{ Child Abuse Score, No. (\%) } & \multirow{2}{*}{$P$ value } \\
\hline & Low & Moderate & High & \\
\hline Child physical abuse & $44(22.6)$ & $32(16.4)$ & $119(61)$ & \multirow{5}{*}{0.000} \\
\hline Child sexual abuse & $49(25.1)$ & $85(43.6)$ & $61(31.3)$ & \\
\hline Malnutrition & $38(19.5)$ & $86(44.1)$ & $71(36.4)$ & \\
\hline Neglect & $24(12.3)$ & $43(22.05)$ & $128(65.65)$ & \\
\hline Child emotional abuse & $34(17.44)$ & $40(20.51)$ & $121(62) .05$ & \\
\hline
\end{tabular}

Analysis was performed by chi-square test.

* The statistically significant level of $P$ value is less than 0.05 . 
of suicide described moderate to severe malnutrition in the current research. A study in 2014 revealed that suicidal cases had significantly lower mean levels of vitamin D than depressed non-suicidal subjects and healthy controls, which could be related to the inflammatory alterations identified in these victims (26). Another investigation examined the association between childhood hunger and repetitive thoughts of death or self-destruction in older adults. It revealed that the consequences of childhood hunger might be long-lasting and correlated with psychic health and well-being, even in older adults (27). In the present study, the emotional separation of parents and divorce had the highest percentage in patients. The results of a meta-analysis in 2019 showed that people affected by parental separation had a greater possibility of exhibiting a classification of mental health diseases (28). Similarly, some researchers demonstrated the relations between adverse childhood experiences and more significant dangers of depression, drug abuse, and smoking that confirm the importance of considering self-destruction and the suicide of these individuals $(29,30)$.

In the current study, neglect and child abuse had the highest prevalence, which could be due to differences in individuals' culture and lifestyles in different locations. Finally, it was concluded that children's sexual abuse should be recognized as a significant risk factor for suicide and self-harm under multiple models. It should also be noted that there is a growing need for comprehensive studies of child abuse.

\section{Conclusion}

The abused child is not equipped with proper behavioral skills, and due to inadequate training, he or she has low self-esteem and self-confidence. Therefore, he or she may commit suicide in adolescent or youth period and even in adulthood in the face of some adversity and failure. It is the responsibility of the community towards the child to inform the parents, educate them, and apply the right culture of child respect. Further investigation is needed to help children affected by separation, neglect, and abuse.

\section{Author's contributions}

Study concept and design: AD and MS. Acquisition of data: ZF. Analysis and interpretation of data: MC. Drafting of the manuscript: $\mathrm{AD}$ and ZF. Critical revision of the manuscript for important intellectual content: AD and MS. Statistical analysis: MC. Administrative, technical, and material support: $M C, A D$ and MS. Study supervision: AS.

\section{Ethical issues}

The Ethics Committee of Ahvaz Jundishapur University of Medical Sciences approved this study by code number IR. AJUMS.REC.1396.1000.

\section{Acknowledgments}

This study is part of a thesis by Zahra Farah bakhsh and was financially supported by the vice-chancellor of research affairs of the Ahvaz Jundishapur University of Medical Sciences. The authors would like to thank Golestan and Imam Khomeini hospitals.

\section{References}

1. Pipe ME, Lamb ME, Orbach Y, Cederborg AC. Child Sexual Abuse: Disclosure, Delay, and Denial. Psychology Press; 2013.

2. Arensman E, Larkin C, McCarthy J, Leitao S, Corcoran P, Williamson E, et al. Psychosocial, psychiatric and workrelated risk factors associated with suicide in Ireland: optimised methodological approach of a case-control psychological autopsy study. BMC Psychiatry 2019; 19(1): 275. doi: 10.1186/s12888-019-2249-6.

3. Page A, Sperandei S, Spittal MJ, Milner A, Pirkis J. The impact of transitions from employment to retirement on suicidal behaviour among older aged Australians. Soc Psychiatry Psychiatr Epidemiol 2021; 56(5): 759-71. doi: 10.1007/s00127-020-01947-0.

4. Chang Q, Yip PSF, Chen Y-Y. Gender inequality and suicide gender ratios in the world. J Affect Disord 2019; 243: 297 304. doi: 10.1016/j.jad.2018.09.032

5. Szanto K, Bruine de Bruin W, Parker AM, Hallquist MN, Vanyukov PM, Dombrovski AY. Decision-making competence and attempted suicide. J Clin Psychiatry 2015; 76(12): e1590-7. doi: 10.4088/JCP.15m09778.

6. MacMillan HL, Tanaka M, Duku E, Vaillancourt T, Boyle $\mathrm{MH}$. Child physical and sexual abuse in a community sample of young adults: results from the Ontario Child Health Study. Child Abuse Negl 2013; 37(1): 14-21. doi: 10.1016/j.chiabu.2012.06.005.

7. Devries KM, Mak JY, Child JC, Falder G, Bacchus LJ, Astbury J, et al. Childhood sexual abuse and suicidal behavior: a meta-analysis. Pediatrics 2014; 133(5): e133144. doi: 10.1542/peds.2013-2166.

8. Bagley C, Mallick K. Prediction of sexual, emotional, and physical maltreatment and mental health outcomes in a longitudinal cohort of 290 adolescent women. Child Maltreat 2000; 5(3): 218-26. doi: 10.1177/1077559500005003002.

9. Tomoda A, Navalta CP, Polcari A, Sadato N, Teicher $\mathrm{MH}$. Childhood sexual abuse is associated with reduced gray matter volume in visual cortex of young women. Biol Psychiatry 2009; 66(7): 642-8. doi: 10.1016/j. biopsych.2009.04.021.

10. Andersen SL, Tomada A, Vincow ES, Valente E, Polcari A, Teicher MH. Preliminary evidence for sensitive periods in the effect of childhood sexual abuse on regional brain development. J Neuropsychiatry Clin Neurosci 2008; 20(3): 292-301. doi: 10.1176/jnp.2008.20.3.292.

11. Ito Y, Teicher MH, Glod CA, Ackerman E. Preliminary evidence for aberrant cortical development in abused children: a quantitative EEG study. J Neuropsychiatry Clin Neurosci 1998; 10(3): 298-307. doi: 10.1176/jnp.10.3.298.

12. Braquehais MD, Oquendo MA, Baca-García E, Sher L. Is impulsivity a link between childhood abuse and suicide? Compr Psychiatry 2010; 51(2): 121-9. doi: 10.1016/j. comppsych.2009.05.003.

13. Laird JJ, Klettke B, Hall K, Clancy E, Hallford D. Demographic and psychosocial factors associated with child sexual exploitation: a systematic review and meta- 
analysis. JAMA Netw Open 2020; 3(9): e2017682. doi: 10.1001/jamanetworkopen.2020.17682.

14. Lim L, Hart H, Mehta M, Worker A, Simmons A, Mirza K, et al. Grey matter volume and thickness abnormalities in young people with a history of childhood abuse. Psychol Med 2018; 48(6): 1034-46. doi: 10.1017/s0033291717002392.

15. Lim L, Hart H, Howells H, Mehta MA, Simmons A, Mirza $\mathrm{K}$, et al. Altered white matter connectivity in young people exposed to childhood abuse: a tract-based spatial statistics (TBSS) and tractography study. J Psychiatry Neurosci 2019; 44(4): E11-E20. doi: 10.1503/jpn.170241.

16. Harford TC, Yi HY, Grant BF. Associations between childhood abuse and interpersonal aggression and suicide attempt among U.S. adults in a national study. Child Abuse Negl 2014; 38(8): 1389-98. doi: 10.1016/j. chiabu.2014.02.011.

17. Afifi TO, MacMillan HL, Boyle M, Taillieu T, Cheung K, Sareen J. Child abuse and mental disorders in Canada. CMAJ 2014; 186(9): E324-32. doi: 10.1503/cmaj.131792.

18. Salokangas RKR, Luutonen S, Heinimaa M, From T, Hietala $\mathrm{J}$. A study on the association of psychiatric diagnoses and childhood adversities with suicide risk. Nord J Psychiatry 2019; 73(2): 125-31. doi: 10.1080/08039488.2018.1493748.

19. Simbar M, Golezar S, Alizadeh S, Hajifoghaha M. Suicide risk factors in adolescents worldwide: a narrative review. J Rafsanjan Univ Med Sci 2018; 16(12): 1153-68. [Persian].

20. Maniglio R. The role of child sexual abuse in the etiology of suicide and non-suicidal self-injury. Acta Psychiatr Scand 2011; 124(1): 30-41. doi: 10.1111/j.1600-0447.2010.01612.x.

21. Yaghoubi Doust $M$, Enayat $H$. Sociological factors of domestic violence towards adolescent female children (case study: high schools in Ahwaz). Woman \& Study of Family 2012; 4(14): 143-71. [Persian].

22. Der EM, Dakwah IA, Derkyi-Kwarteng L, Badu AA. Hanging as a method of suicide in Ghana: a 10 year autopsy study. Pathol Discov 2016; 4: 2. doi: 10.7243/2052-7896-4-2.

23. Asscher JJ, Van der Put CE, Stams GJ. Gender differences in the impact of abuse and neglect victimization on adolescent offending behavior. J Fam Violence 2015; 30(2): 215-25. doi: 10.1007/s10896-014-9668-4.

24. Higgins DJ, McCabe MP. Multiple forms of child abuse and neglect: adult retrospective reports. Aggress Violent Behav 2001; 6(6): 547-78. doi: 10.1016/s1359-1789(00)00030-6.

25. Vanderminden J, Hamby S, David-Ferdon C, KachaOchana A, Merrick M, Simon TR, et al. Rates of neglect in a national sample: child and family characteristics and psychological impact. Child Abuse Negl 2019; 88: 256-65. doi: 10.1016/j.chiabu.2018.11.014.

26. Grudet C, Malm J, Westrin A, Brundin L. Suicidal patients are deficient in vitamin $\mathrm{D}$, associated with a pro-inflammatory status in the blood. Psychoneuroendocrinology 2014; 50: 210-9. doi: 10.1016/j.psyneuen.2014.08.016.

27. Stickley A, Koyanagi A, Inoue Y, Leinsalu M. Childhood hunger and thoughts of death or suicide in older adults. Am J Geriatr Psychiatry 2018; 26(10): 1070-8. doi: 10.1016/j. jagp.2018.06.005.

28. Auersperg F, Vlasak T, Ponocny I, Barth A. Long-term effects of parental divorce on mental health - a metaanalysis. J Psychiatr Res 2019; 119: 107-15. doi: 10.1016/j. jpsychires.2019.09.011.

29. Léveillée S, Doyon L, Cantinotti M. "Evolution of paternal filicide-suicide in the province of Quebec". Encephale 2019; 45(1): 34-9. doi: 10.1016/j.encep.2017.10.007. [French].

30. Houtepen LC, Heron J, Suderman MJ, Fraser A, Chittleborough CR, Howe LD. Associations of adverse childhood experiences with educational attainment and adolescent health and the role of family and socioeconomic factors: a prospective cohort study in the UK. PLoS Med 2020; 17(3): e1003031. doi: 10.1371/journal.pmed.1003031. 